\title{
Algorithm for automated calculation of a segmented electromechatronic module of robotic system motion
}

\author{
Mikhail Shepelenko* and Roman Meshcheryakov \\ Tomsk State University of Control Systems and Radioelectronics, Lenina str. 40, 634050 Tomsk, Russia
}

\begin{abstract}
The paper deals with the design of digital models of the elements of the electromechatronic part of motion module of robotic systems, which provides increasing productivity of the electromechatronic components of robotic and mechatronic manipulators. The developed software provides 3D-modeling of the electromechatronic part; calculation of electromechatronic and electrical elements and visualization of the electrical machine part. It reduces the cost of designing electromechatronic components of manipulators of robotic and mechatronic systems.
\end{abstract}

\section{Introduction}

Modern studies of the creation of electromechatronic modules of motion (EMM) of robotic systems with a segment inductor (inductors) have shown that it is possible to automate development of the EMM assortment range quickly and with a minimum of labor. In modern conditions, when choosing computer-aided design (CAD) systems, users are increasingly paying attention to the possibilities provided by the system in the field of parameterization, and enterprise managers already imply not only the transfer of the process from manual production of drawings to the computer.

The requirements for modern computer-aided design systems are not limited to the convenience of creating graphic primitives and drawing up drawings in accordance with GOST; on the agenda there appear the capabilities of systems in the field of constructing associative models and assemblies; the degree of simplicity in creating their own parametric libraries is estimated; Use the enterprise's existing know-how. Today, designing on the basis of existing blanks (design based on knowledge bases) makes it possible to significantly reduce the time of the project and the number of errors, which often plays a decisive role in a highly competitive environment.

A range of electromechatronic modules of motion has been developed by Elektromechatronic Systems LLC: segmental, arc and linear EMM, which are the basic elements of a gearless electric drive that rotates along an arc or a circle, as well as linear movements of the operating elements of various devices and mechanisms (Fig. 1).

The research task was to develop modern methods of automated calculation, since the main requirement in the modernization of industry and import substitution is the accelerated design of robotic and mechatronic systems for products used in such spheres as engineering, medicine and radio electronics [1-7].

\section{Parametric modeling}

Methods of parametric modeling (design) and prototyping in CAD, PDM-systems were considered. Precisely parameterization allows solving constructive schemes and variants in a short time, which in future will help avoid fundamental mistakes in the design process by changing variables in functions, parameters and geometrical relationships. Parametric modeling is significantly different from 3D modeling. In the case of parametric design, the designer creates a mathematical model of elements with parameters that change the configuration of the part, the mutual movement of parts in the assembly, etc [8-10].

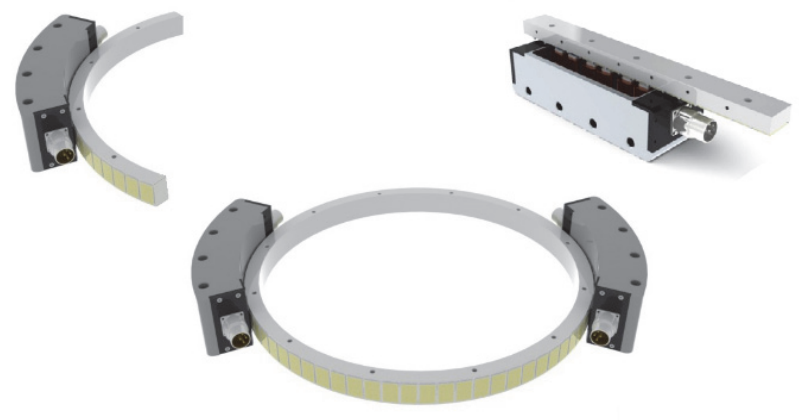

Fig. 1. EMM assortment range.

The idea of parametric modeling appeared at the early stages of the development of CAD systems, but for a while it could not be implemented because of insufficient computer capacities in those years. The first known CAD with the possibility of parameterization came out in 1989. Pioneers were Pro / Engineer (threedimensional solid-state parametric modeling) of

\footnotetext{
* Corresponding author: mixan_19@mail.ru
} 
Parametric Technology Corporation and T-FLEX CAD (two-dimensional parametric modeling) of Top Systems.

There are several types of parameterization: tabular, hierarchical, variational (dimensional) and geometric. In our case, all types are involved. But for the most part variational and geometric parameterization. Because parameterization is based on the construction of sketches (with overlapping of various parametric links on the sketch objects) and the imposition of constraints by the user in the form of a system of equations that determine the relationships between the parameters. This in its turn makes it possible to easily change the shape of the sketch or the size of the parameters of operations; this makes it convenient to modify the digital model. As for the geometric, in it the geometry of each parametric element is recalculated depending on the position of the parent objects, its parameters and variables. It should be noted that geometric parameterization allows for more flexible editing of models. If there is a need to make an unplanned change, then it is not necessary to delete the original construction lines in the geometry of the model. After the design concept is selected, the designer begins to create the original model of the product. Here, along with parameterization, another invention, solid-state modeling, comes to its aid. The basic idea of solid-state modeling is to always guarantee a physically consistent representation of geometric volumetric bodies and to operate with the concepts of adding / removing material. A correct solid body contains an internal volume bounded by the outer surface of the body. This representation allows you to determine the body volume, its mass, moments of inertia, center of gravity, etc. These parameters are often critical in evaluating the design effectiveness of the product and are extremely important for the designer [11-13].

Based on the method of parametric construction of the model of the elements of the EMM, it was decided to develop a subsystem for CAD Autodesk Inventor for the automated calculation of the segmented EMM (SEMM). In it, it will be necessary to work out the algorithm of interaction of the subsystem itself with $\mathrm{CAD}$, and also to envisage and develop each stage of creation of all elements that are part of the EMM.

\section{Generation of element parameters of EMM}

The developed program "Automated calculation of the product range of the electromechatronic part of electromechatronic modules of motion" is operated in Windows 7/8 x64 with Autodesk Inventor 2014 installed. The software package is developed in the language of Visual Basic .NET (VB.NET). The program is designed to interact with CAD Autodesk Inventor 2014. Input data are models. Reference models are parts and assemblies made in Autodesk Inventor 2014 using the Ilogic tool.

The models consist of 6 parts: the inductor case, the coil, the covers, the magnet, the rotor housing, the rotor magnet and four assemblies: the inductor, the rotor magnet core, the rotor and the SEMM calculation of the product range of the electromechatronic part of electromechatronic motion modules" designed to increase the degree of automation of the design of EMM for manipulators of robotic systems. The subsystem of the automated calculation of the electromechatronic part extends the functionality of the basic CAD Autodesk Inventor - a system of three-dimensional solid and surface parametric design, designed to create digital prototypes of industrial products and provide: the calculation of electrical elements and automatic input to the basic CAD Autodesk Inventor algorithms for calculation of electromechatronic parts with inductor for $3 \mathrm{D}$ visualization of the layout of electromechatronic parts (Fig. 2).

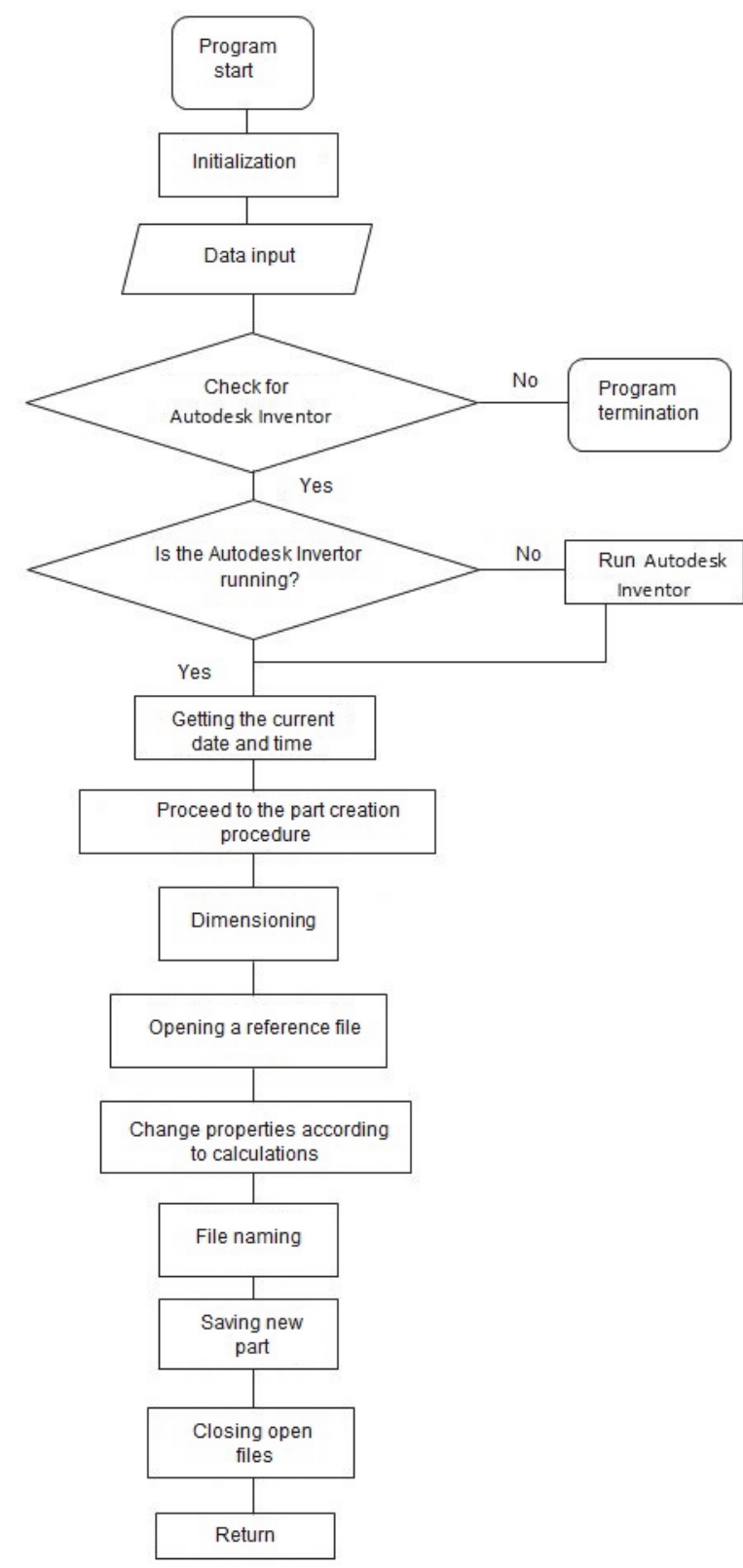

Fig. 2. The block diagram of the program.

The main function of the program is the generation of 3D models of parts and assemblies of parts of electromechatronic motion modules within a given range of products. The program starts with the launch of the 
MyInventorPlug-in.exe program file. When the program starts, the main program window is displayed (Fig. 3).

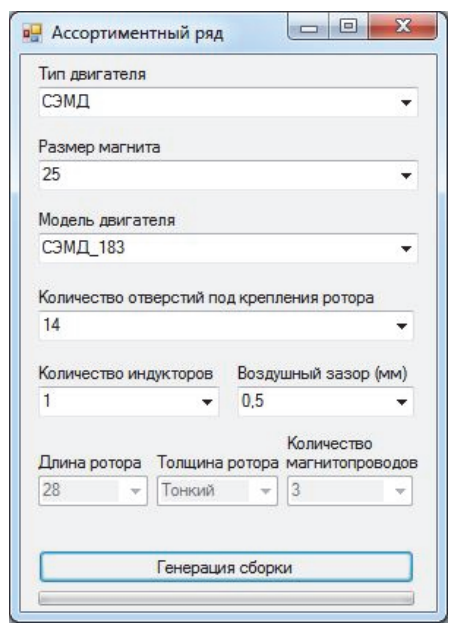

Fig. 3. The main program window.

To generate parts and assemblies of the range of the electromechatronic part of the electromechatronic motion module, the user can first select a number of parameters for generating. To do this, in the "Magnet size" field from the drop-down list, you must select one of the size values for the magnet: 25 or 50 . Next, in the "Engine type" field, you can select the appropriate engine type from the drop-down list. The program will automatically set the necessary geometric parameters corresponding to the selected type. If the user did not select anything or chose not all values, the default values are used as the remaining values. To generate details and assemblies with the set values, the user should click the "Generate assembly" button [1].

For example, consider the creation of an inductor housing SEMM. Figures 4 and 5 show the window of the parameters of the functions of the inductor housing of the SEMM and the digital model of the inductor housing.

\subsection{Generation of the inductor housing}

Part generation occurs in the CreateInductor () procedure (Fig. 6). Based on the user-selected magnet size, the actual size is calculated. Then, a reference sketch of the inductor case is opened (all reference sketches are stored in a folder named InvModel). From the array of standard sizes, the dividing and outer diameters of the inductor housing are programmed programmatically. The internal diameter of the inductor case corresponds to the divisor diameter, increased by 0.5 .

A sketch of the inductor case with the required dimensions is created by software making changes to the master sketch: the parameter that is responsible for the height of the inductor case, and the previously dimensioned height is assigned, and the required external and internal diameters are assigned to the sketch. Then, the received sketch of the inductor case is saved with the file name assigned to it in the format: Part name type of the engine the current date on this computer in the format yyyy.MM.dd-HH.mm.ss. The file is assigned the .ipt extension.

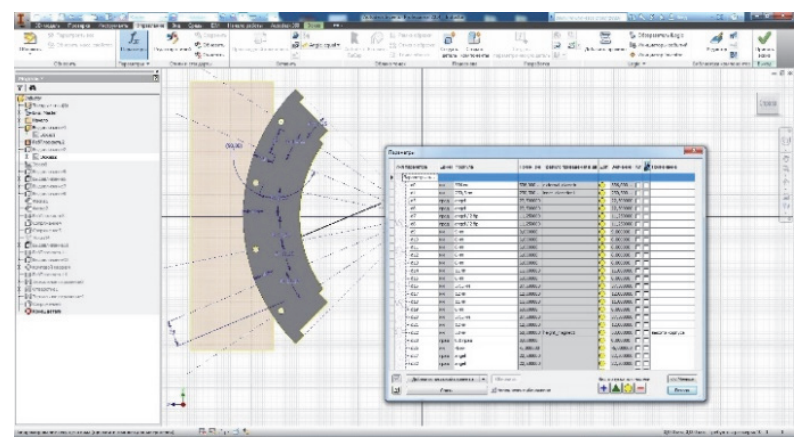

Fig. 4. Creating inductor housing.

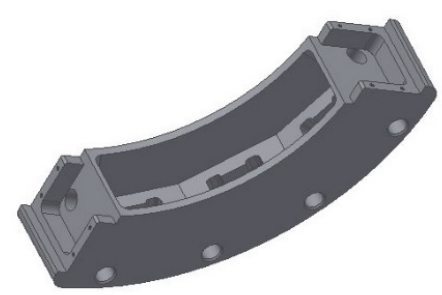

Fig. 5. Inductor housing.

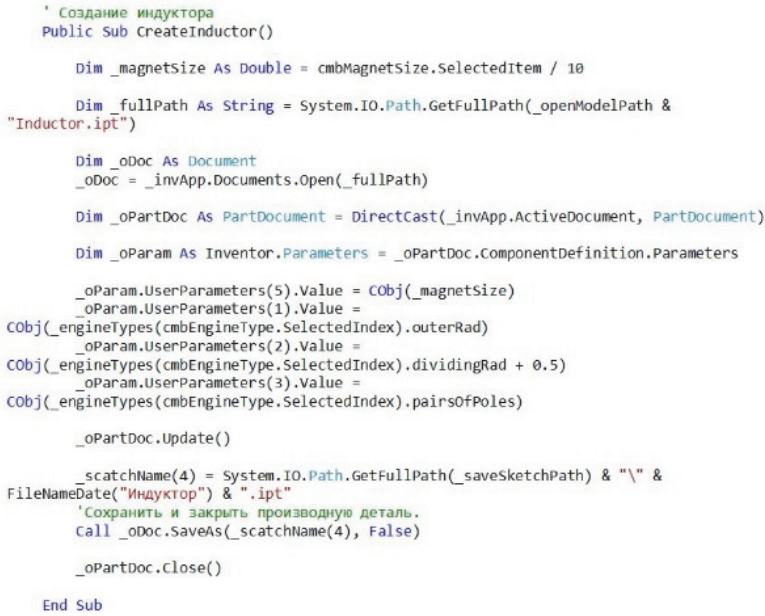

Fig. 6. Fragment of the program listing.

\subsection{Generation of the steel ring of rotor}

Part generation occurs in the CreateRotor () procedure. Based on the size of such a large number of rotor rings. From the array of standard sizes, the dividing and internal diameters of the rotor are programmed programmatically. The outer diameter of the rotor corresponds to the dividing diameter, reduced by 0.5 . Then the reference sketch of the rotor is opened (all reference sketches are stored in a folder named InvModel). A sketch of the rotor with the required dimensions for making changes to the master sketch: to obtain the required size and the required size, as well as the required outer and inner diameters. Number of holes for fastening. Now it stores the data in a format. The file is assigned the .ipt extension. The file is saved in the folder with the name SEMD_size of the engine । thumbnails (Fig. 7). 


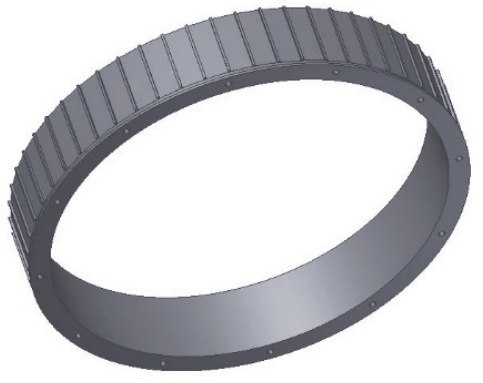

Fig. 7. The steel ring of rotor.

After the generation of all the details has ended, the generation of assemblies of the SEMM that are part of the main assembly begins, such as: the rotor, the inductor magnet core, the inductor and the SEMM itself.

\subsection{Generation of the rotor}

Generation of the assembly occurs in the procedure CreateAssemblyRotor (). Opens the master assembly of the rotor, containing links to the sketches of the rotor and magnet (all reference assemblies are stored in a folder called InvModel). The reference to the assembly elements containing the rotor and magnet sketches is assigned new values from the array _scatchName, after which the corresponding assembly elements begin to reference the newly created sketches. Then the parameter responsible for the number of magnets on the rotor is assigned the current value corresponding to the number of magnets for this type of motor. The resulting rotor assembly is saved with the corresponding file name assigned to it (Fig. 8). The file is assigned the extension iam. The file is saved in the folder with the name SEMM_size engine $\backslash$ Assemblies.

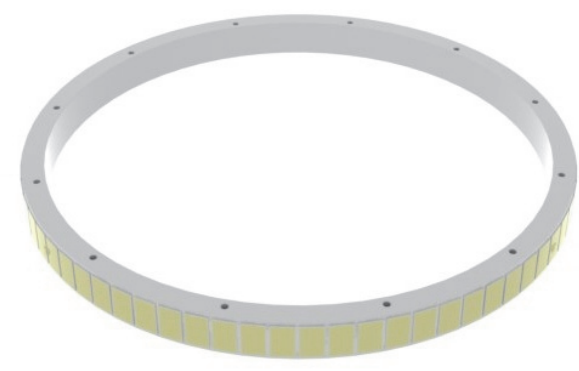

Fig. 8. Rotor SEMM.

\subsection{Generation of the magnetic core of the inductor}

Generation of the assembly occurs in the procedure CreateAssemblyMagnetInductor (). A reference assembly of the inductor magnetic core is opened, containing references to the coil and core sketches (all reference assemblies are stored in a folder named InvModel). A reference to the assembly elements containing coil and core sketches is assigned new values from the array scatchName, after which the corresponding assembly elements begin to reference the newly created thumbnails. The obtained assembly of the magnetic circuit is stored and assigned the extension .iam. The file is saved in the folder with the name SEMM_size engine \Assemblies. The name of the assembly files is stored in the assemblyName data set for later use.

\subsection{Inductor generation}

Assembly generation occurs in the CreateAssemblyInductor () procedure. A reference assembly of the inductor is opened, containing references to the sketch of the inductor case, the cover of the magnetic coil and to the assembly of the inductor magnetic core (all reference assemblies are stored in a folder named InvModel). The reference to the assembly elements containing the inductor body and the rotor magnet lead are assigned new values from the _assemblyName array, after which the corresponding assembly elements begin to reference the newly created models (Fig. 9).

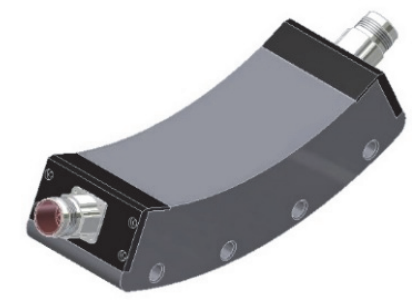

Fig. 9. SEMM inductor.

The obtained inductor assembly is saved with the file name assigned to it in the format: Assembly name type of the engine the current date on this computer in the format yyyy.MM.dd-HH.mm.ss. The file is assigned the extension .iam. The file is saved in the folder with the name SEMM_size engine $\backslash$ Assemblies. The assembly file name is stored in the _assemblyName data set for later use.

\subsection{Generation of SEMM}

Assembly generation occurs in the procedure CreateAssemblySEMM (). The reference assembly of the SEMM is opened, containing references to the inductor and rotor assemblies (all reference assemblies are stored in a folder named InvModel). A reference to the assembly elements containing the inductor and the rotor are assigned new values from the array _assemblyName, after which the corresponding assembly elements begin to reference the newly created assemblies. After that, the obtained ECM assembly is saved with the appropriate file name assigned to it. The assembly file name is stored in the _assemblyName data set for later use (Fig.10). 


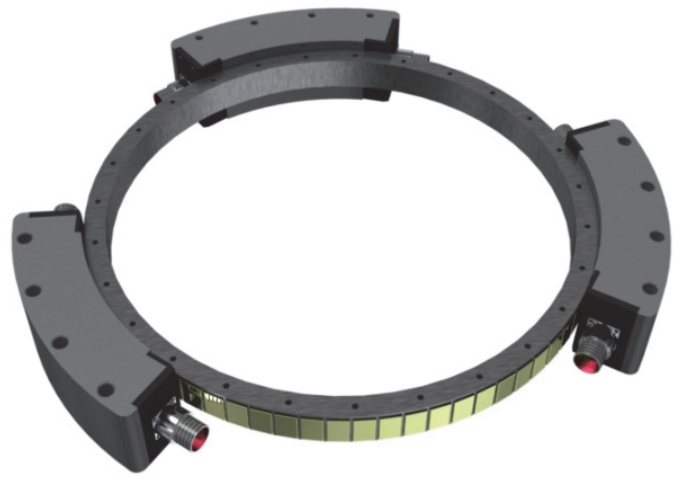

Fig. 10. The main assembly of SEMM.

After executing these procedures, the program waits for further user actions. The user can select new parameters or leave the previous ones and regenerate all the parts and assemblies or can close the program.

\section{Conclusion}

The software designed for calculating the assortment of EMM elements is developed. The program allows you to specify the input data and calculate the optimal values of the elements of the EMM at the output. User input of information about the initial parameters of the projected electromechatronic parts is provided by the interface module, which allows the user to create assembly structures. The program allows for: 3D-modeling of the electromechatronic part; calculation of electromechatronic and electrical elements and visualization of the electrical machine part; reduction of the cost of designing electromechatronic components of manipulators of robotic and mechatronic systems. The program allows subsequent docking with CAD Autodesk Inventor Professional to automatically obtain design documentation for ESKD.

\section{References}

1. L. Chubraeva, A. Ronzhin, A. Shyshlakov, Al. Ronzhin and V. Shyshlakov, SPIIRAS Proc., 33(2), 207-226 (2014)

2. O.Yu. Osipov, Yu.M. Osipov, S.V. Shcherbinin, Multicoordinate electromechatronic motion systems: monograph, Tomsk: Publishing house TUSUR, - p. 320 (2010)

3. General idea of CAD Autodesk Inventor [Electronic resource]. - Access mode: http://www.autodesk.ru/adsk/servlet/index?siteID=8 71736\&id $=22741018$

4. Yu.M. Osipov, P.K. Vasenin, D.A. Medvedev, Reports of TUSUR, 1(17) (2008)

5. Yu.M. Osipov, B.Ya. Vologdin, Reports of TUSUR, 2(22) (2010)

6. Yu.M. Osipov, S.V. Shcherbinin, Mechatronics, automation, management. 8 (2012)

7. I. Kalyaev, A. Dordopulo, I. Levin, A. Fedorov, SPIIRAS Proc., 50(1), 5-31 (2017)

8. Using the PDM-system in the design of technological processes [Electronic resource]. Access http://de.ifmo.ru/bk_netra/page.php?dir=2\&tutindex $=4 \&$ index $=54 \&$ layer $=1$

9. A.G. Kiselev, Project management for the development and implementation of information systems (PMI): a textbook, for undergraduates. (Novosibirsk, 2010)

10. M.G. Shepelenko, A.N. Lunin, Scientific session of the TUSUR. Materials of the reports of the AllRussian scientific and technical conference of students, graduate students and young scientists, Tomsk, - Tomsk, V-Spectrum (2008)

11. M.G. Shepelenko, Scientific session of TUSUR. Materials of the reports of the All-Russian scientific and technical conference of students, graduate students and young scientists, Tomsk, V-Spectrum, (2010)

12. E. Yares, Design World. WTWH Media, LLC. 8(4) (2013)

13. N. Golovanov, Geometric Modeling: The mathematics of shapes. (CreateSpace Independent Publishing Platform 2014) 